\title{
SAGE detects microRNA precursors
} Xijin $\mathrm{Ge}^{1}$, Qingfa $\mathrm{Wu}^{1}$ and San Ming Wang*1,2

\author{
Address: ${ }^{1}$ Center for Functional Genomics, Division of Medical Genetics, Department of Medicine, ENH Research Institute, 1001 University Place, \\ Evanston, IL 60201 USA and ${ }^{2}$ Robert H. Lurie Comprehensive Cancer Center, Northwestern University Feinberg School of Medicine, 1001 \\ University Place, Evanston, IL 60201 USA \\ Email: Xijin Ge - xge@northwestern.edu; Qingfa Wu - qwu@enh.org; San Ming Wang* - swang1@northwestern.edu \\ * Corresponding author
}

Published: 07 November 2006

BMC Genomics 2006, 7:285 doi:10.1 |86/|47|-2/64-7-285
Received: 19 July 2006

Accepted: 07 November 2006

This article is available from: http://www.biomedcentral.com/l47/-2164/7/285

(c) $2006 \mathrm{Ge}$ et al; licensee BioMed Central Ltd.

This is an Open Access article distributed under the terms of the Creative Commons Attribution License (http://creativecommons.org/licenses/by/2.0), which permits unrestricted use, distribution, and reproduction in any medium, provided the original work is properly cited.

\begin{abstract}
Background: MicroRNAs (miRNAs) have been shown to play important roles in regulating gene expression. Since miRNAs are often evolutionarily conserved and their precursors can be folded into stem-loop hairpins, many miRNAs have been predicted. Yet experimental confirmation is difficult since miRNA expression is often specific to particular tissues and developmental stages.

Results: Analysis of 29 human and 230 mouse longSAGE libraries revealed the expression of 22 known and 10 predicted mammalian miRNAs. Most were detected in embryonic tissues. Four SAGE tags detected in human embryonic stem cells specifically match a cluster of four human miRNAs (mir-302a, b, c\&d) known to be expressed in embryonic stem cells. LongSAGE data also suggest the existence of a mouse homolog of human and rat mir-493.
\end{abstract}

Conclusion: The observation that some orphan longSAGE tags uniquely match miRNA precursors provides information about the expression of some known and predicted miRNAs.

\section{Background}

MicroRNAs (miRNAs) are endogenous, 22 nucleotide (nt) noncoding RNAs that play important roles in gene expression regulation by base-pairing with messenger RNAs [1]. A single miRNA can down-regulate a large number of target mRNAs [2]. Since most miRNA precursors can be mapped to 60-120 nt long conserved genomic regions and can be folded into hairpin structures, miRNAs can be predicted from genomic sequences with high sensitivity [3-9]. Experimental confirmation and functional analysis of these predicted miRNAs, however, remains a challenge.

Serial analysis of gene expression (SAGE) collects short 14-21 nt tags from 3' ends of transcripts after certain restriction enzyme cutting sites; the most frequently used site is "CATG" which is recognized by NalIII [10] recently developed variation of this technique known as longSAGE collects 21 bp tags, which are long enough for genomic mapping and specific annotation [11]. Unlike DNA microarray that depends on a pre-defined gene set, SAGE is an exploratory method for transcriptome analysis. Many orphan SAGE tags that cannot be associated with any known transcripts represent potential novel transcripts [12].

Primary miRNAs transcribed by polymerase II are processed by the nuclear Drosha enzyme to give pre-miRNAs, which are then exported into cytoplasm and lead to mature miRNAs. At least some primary miRNAs are known to be capped and polyadenylated in the nucleus [13]. As recent analysis of EST identified 26 known miR- 
NAs [14], SAGE might also be able to detect some primary miRNAs. To investigate whether this is the case, we mined the large number of human and mouse longSAGE tags deposited in public databases and compared these tags with the sequences of pre-miRNAs.

\section{Results and discussion}

To identify a set of SAGE tags that could theoretically be contributed by miRNAs, we searched for "CATG" sites in known miRNA precursors. Among the 332 known human miRNAs in the miRBASE [15], 92 (28\%) bear such sites. Similarly, $64(24 \%)$ of the 270 known mouse miRNAs could contribute to SAGE tags. To increase coverage, we also included longSAGE tags uniquely mapped to genomic loci that are very close (within $30 \mathrm{bp}$ ) to known hairpin sequences. This is because the complex process of miRNA biogenesis is still not well understood and the complete primary transcription units, which can be significantly longer than the $\sim 60-120$ bp hairpin sequence, have not been defined for most miRNAs. After extension, the number of human and mouse miRNAs associated with longSAGE tags increased to 130 (39\%) and 99 $(37 \%)$, respectively. Thus, SAGE can theoretically detect about one-third of known miRNAs. Additional File 1 lists all these miRNAs and corresponding longSAGE tags.

These virtual tags were then compared with experimentally observed tags in 29 human and 120 mouse longSAGE libraries in the Gene Expression Omnibus database [16] and in 110 mouse longSAGE libraries representing various tissues in multiple developmental stages from the Mouse Atlas of Gene Expression website [17]. We identified nine longSAGE tags matched to human miRNAs and 16 matched to mouse miRNAs. These tags were then mapped to human or mouse genomic sequences and annotated with available mRNAs and ESTs. After removing tags that may have originated from known genes (e.g., mapping to the sense strand of an exon including UTR) and those that mapped to multiple genomic loci, we identified eight human and 14 mouse longSAGE tags that represent known miRNAs (Table 1).

Among the eight human miRNAs whose expression was detected by SAGE tags, four (mir-302a, b, c\&d) mapped to a 600 bp region of Chr. 4q25 (Fig. 1). Another member of the cluster, mir-367, was not detected because of the lack of the "CATG" site. This miRNA cluster is known to be specifically expressed in human embryonic stem cells [18], which is in accord with the source of the SAGE libraries in which the tags were observed (see Table 1, detailed information about SAGE libraries is available in Additional File 2).

The large amount of mouse longSAGE data provides rich information about the particular tissue and developmen- tal stage of the expression of 14 known miRNAs. In the mouse embryo at Theiler Stage 14, for example, we observed the expression of mir-133a-2 and mir-351 in heart ventricle. At the same stage, SAGE detects the expression of mir-29b-2 in heart bulbous cordis. The expression of mir-29b and mir-133 in the heart has been confirmed by northern blot [19].

LongSAGE data also indicate the expression of "known" but unconfirmed miRNAs, such as the expression of let-7i in human embryonic stem cells and fetal brain tissues. Although listed as known miRNAs in the miRBASE [15] based on the mouse homolog, its expression has not yet been experimentally confirmed in humans. Similarly, longSAGE tags also suggest the expression of two human (mir-7-1 and mir-125a) and three mouse (mir-331, mir351, and mir-495) miRNAs that have not been experimentally confirmed (Table 1). LongSAGE data thus provide hints about the expression of unconfirmed miRNAs.

LongSAGE data also provide evidence for the existence of some predicted miRNAs. Two human and seven mouse miRNAs predicted by Lim et al. [4], Berezikov et al. [7] and Sewer et al. [9] are supported by SAGE tags (Table 1). One mouse miRNA candidate, cand202-MM, predicted by both Berezikov et al. [7] and Sewer et al. [9], is highly homologous to human and rat mir-493. The presence of such a SAGE tag in two mouse SAGE libraries strongly supports the existence of mouse mir-493. Two mouse SAGE tags map to genomic loci that are highly homologous to predicted human (cand847-HS) and rat (cand913-RN) miRNAs. The information about the tissue and stage of expression might facilitate the experimental confirmation of these predicted miRNAs.

The use of SAGE tags to detect miRNA precursors is limited, however. For example, longSAGE tags are subject to sequencing errors. Also, 21 bp tags do not provide full sequences of miRNA precursors. Therefore, further studies are needed to confirm our findings.

\section{Conclusion}

In summary, the available longSAGE tags indicate the expression of eight human and 14 mouse known miRNA precursors and provide evidence for the existence of two human and seven mouse predicted miRNAs. Although limited in the number of miRNAs, SAGE data provide useful information on the expression of miRNA. Together with recent longSAGE-based studies that identifies many novel antisense transcripts in mouse [21] and human [22], this study again shows that longSAGE is an effective technology for exploratory transcriptome analysis. 
Table I: LongSAGE tags matched to known and predicted miRNA precursors.

\begin{tabular}{|c|c|c|c|c|c|c|}
\hline miRNA (1) & longSAGE tags (2) & Chr. & EST & \#libs & Tag counts & Tissue (mouse Theiler Stage) \\
\hline \multicolumn{7}{|c|}{ Human SAGE tags matched to known miRNAs } \\
\hline hsa-mir-302a & TTTTGGTGATGGTAAGT & $4 q 25$ & No & 1 & I & Embryonic stem cell \\
\hline hsa-mir-302b (3) & GAAGTGCTTTCTGTGAC & $4 q 25$ & Yes & 5 & 9 & Embryonic stem cell \\
\hline hsa-mir-302c & TTTCAGTGGAGGTGTCT & $4 q 25$ & Yes & 1 & 2 & Embryonic stem cell \\
\hline hsa-mir-302d & TTTGAGTGTGGTGGTTC & $4 q 25$ & No & 4 & 6 & Embryonic stem cell \\
\hline hsa-mir-7-I (4) & ССТСТACAGGACAAATG & $9 q 21$ & No & 3 & 3 & White blood cell, breast tumor, stem cell \\
\hline hsa-let-7i (4) & GCCCTGGCTGAGGTAGT & $12 q 14$ & No & 4 & 4 & Embryonic stem cells and Fetal brain \\
\hline hsa-mir-2I & GCTGTACCACCTTGTCG & $17 q 23$ & Yes & 2 & 2 & White blood cell, breast tumor \\
\hline hsa-mir-125a (4) & TTGCCAGTCTCTAGGTC & $19 q 13$ & No & 1 & I & breast tumor (myofibroblast) \\
\hline \multicolumn{7}{|c|}{ Human SAGE tags matched to predicted miRNAs } \\
\hline Lim et al. [4] & СTACTСTCACTGAGTAC & $5 p 21$ & No & 1 & & Embryonic stem cell \\
\hline cand525-HS & CGGAGCCCCCGGGCTTG & $1|q| 3$ & No & 4 & & Embryonic stem cell and breast \& lung cancer \\
\hline \multicolumn{7}{|c|}{ Mouse SAGE tags matched to known miRNAs } \\
\hline mmu-mir-29b-2 (3) & GTGGCTTAGATTTTTCC & $\mathrm{IqH} 6$ & Yes & 2 & 2 & Heart bulbous cordis (TSI 4 embryo) \\
\hline mmu-mir-205 & GAGCTGCCAGCGGTGGA & $\mathrm{IqH} 6$ & Yes & 7 & 17 & Brain, forelimb \& skin (embryo) \\
\hline mmu-mir-130a & CCTTTGCTGCTGGCCGG & $2 q D$ & Yes & 1 & 1 & Branchial Arch embryonic tissue \\
\hline mmu-mir-133a-2 & GCCCAGCCAGAGGACAC & $2 \mathrm{qH} 4$ & Yes & 4 & 4 & Heart ventricle (TSI4-19), Sk. muscle (TS25) \\
\hline mmu-mir-29a & ACСTCTTGTGACСССTT & $6 q \mathrm{q} 3$ & No & 1 & I & Ovary (2l days post natal) \\
\hline mmu-mir-425 & GAAAGTGCTTTGGAATG & $9 \mathrm{qF} 2$ & Yes & 3 & 8 & Visual cortex(27days post natal), Pancreas (TS20) \\
\hline mmu-mir-33 I(4) & CAAGCTGAAAGCACTCC & $10 q C 2$ & Yes & 1 & 1 & Brain - Amygdala (Post natal day 7) \\
\hline mmu-let-7i & GCCCTGGCTGAGGTAGT & 10qD2 & Yes & 4 & 4 & Sm. Intestine (TS24), Lung (TS26), Neural tube(TSI3), Pancreas \\
\hline mmu-mir-2l & GCTGTACCACCTTGTCG & $\mathrm{IIqC}$ & Yes & 2 & 2 & Placenta (TS22), large intestine (TS24) \\
\hline mmu-mir-196a-I & GCAGTTACTGCTTCTTG & IIqD & No & 3 & 3 & Endoderm (Definitive), kidney (TS24), testis \\
\hline mmu-mir-337 (3) & CAGGAGTTGATTGCACA & $|2 q F|$ & No & 1 & I & Adrenal gland (TS22 embryo) \\
\hline mmu-mir-485 (4) & TGTGATACTTGGAGAGA & $|2 q F|$ & No & 1 & I & Skin (TS2I) \\
\hline mmu-mir-35I (3,4) & GCACCTCCGTTTCCCTG & $\mathrm{XqA} 5$ & Yes & 2 & 2 & Heart ventricle (TSI4 embryo) \\
\hline mmu-mir-92-2 & CCCATTCATCCACAGGT & $\mathrm{XqA5}$ & No & 1 & 1 & Thymus (TS23 embryo) \\
\hline \multicolumn{7}{|c|}{ Mouse SAGE tags matched to predicted miRNAs } \\
\hline candI85-MM & TGACTGCCTGTCTGTGC & $\mathrm{IqH} 2$ & Yes & 1 & I & Fibroblast cell line (embryonic) \\
\hline cand847-HS & GTGAGCAGATGATGCAT & $2 \mathrm{qHI}$ & Yes & 1 & I & Brain - Whole (TS20 embryo) \\
\hline candI36-MM & AACATTATTTCTTGTGT & $4 q D 2$ & No & 1 & I & Adult testis \\
\hline cand407-MM & GAGTCTTCCAAGCCAAG & $4 \mathrm{qF} 4$ & No & 3 & 3 & Adult bladder \& mammary gland \\
\hline cand9|3-RN & TGACGTCTGAGGAGCGG & I IqE2 & Yes & I & I & Brain telencephalon dorsal (TS20 embryo) \\
\hline cand219-MM & GCCAATCTCCTTTCGGC & $|2 q F|$ & Yes & 2 & 6 & Visual cortex (27 days post natal) \\
\hline cand202-MM & GTAGGCTTTCATTCATT & $|2 q F|$ & No & 2 & 2 & Branchial arch embryonic tissue (TSI5) \\
\hline cand239-MM & CAGCTTTGGAGACGCCA & $16 q C 3$ & No & 1 & 1 & Brain - Preplate (TS2I embryo) \\
\hline cand525-MM & CGGAGCCCCCGGGCTTG & $19 q A$ & No & 9 & 9 & Multiple normal tissues \\
\hline
\end{tabular}

(1) Except the one marked as Lim et al. [4], all predicted miRNAs are based on Berezikov et al. [7]. Cand2 19-MM and Cand202-MM are also predicted by Sewer et al. [9].

(2) "CATG" sites before each SAGE tags had been omitted.

(3) SAGE tags match miRNA hairpin sequences without extension.

(4) These miRNAs are listed as known in miRBASE based on their homolog to entries in other species.

\section{Methods}

Genomic coordinates of 332 human and 270 mouse hairpin sequences were downloaded from the miRBase (Ref. 15 ) as our collection of known miRNAs. Because pre-miRNAs could be longer than these hairpin sequences, these sequences were extended by $30 \mathrm{bp}$ in both directions on corresponding genomic sequences. In addition, miRNAs predicted by Lim et al. [4], Berezikov et al. [7] and Sewer et al. [9] were downloaded from the respective journal web sites. These sequences were then searched for the "CATG" site and 17 bp tags after each of these sites was extracted. Such virtual SAGE tags are linked to miRNAs for further analysis.

The 29 human and 120 mouse longSAGE libraries were retrieved from the gene expression omnibus database
(Ref. 16). Another 110 mouse longSAGE libraries were downloaded from the Mouse Atlas of Gene Expression web site (Ref. 17). Pooling multiple libraries for each species led to a total of 632,813 unique human tags and $1,902,036$ unique mouse tags. These experimental tags were then compared to the virtual tags extracted from miRNA sequences. Only virtual tags whose sequence is identical to the sequence of real tags were considered confirmed.

For annotation, matched human and mouse tags were mapped to human (Mar. 2006 assembly, hg18) and mouse (Aug. 2005 assembly, mm7) genomic sequences, respectively, using BLAT [20]. All tags mapped to multiple genomic loci or exons of known genes were excluded. 


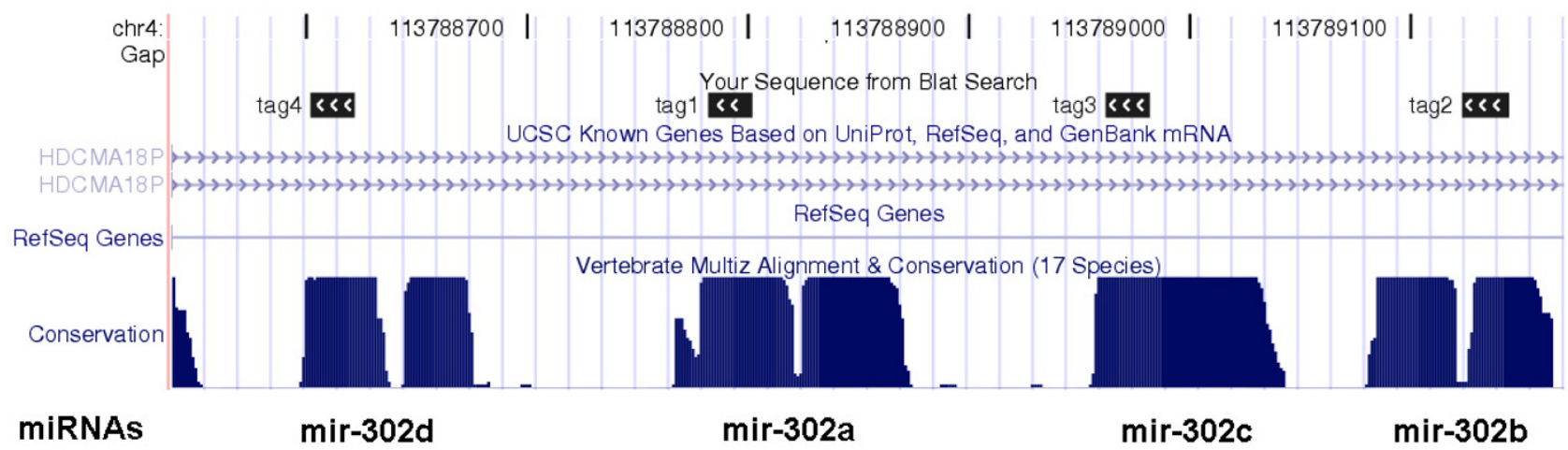

Figure I

Four human longSAGE tags specifically mapped to a cluster of four miRNAs on Chromosome 4. These evolutionarily conserved miRNAs are transcribed from the antisense strand of an intron of HDCMAI8P gene.

Tags mapped to UTR regions were retained only if the tag was transcribed from the opposite strand.

\section{Authors' contributions}

XG, QW and SMW conceived the study and participated in study design. XG did computational analyses. XG and SMW wrote the manuscript. All authors read and approved the final manuscript.

\section{Additional material}

\section{Additional File 1}

Virtual SAGE tags extracted from known miRNA precursors. This file contains 310 longSAGE tags extracted from known miRNA precursor sequences.

Click here for file

[http://www.biomedcentral.com/content/supplementary/1471-

2164-7-285-S1.xls]

\section{Additional File 2}

Detailed description of SAGE libraries that includes tags representing miRNAs precursors. This file gives detailed information on the type of tissue and stage of development (if available) that the SAGE tags listed in Table 1 are detected. SAGE library IDs are also given for further enquiry to the original databases.

Click here for file

[http://www.biomedcentral.com/content/supplementary/14712164-7-285-S2.xls]

\section{Acknowledgements}

The authors thank Wendy Rubinstein for discussion and support, Wei Wu for stimulating discussion, and W. James Kent for suggestions on the usage of BLAT program. This study was supported by the National Institute of Health (to SMW), US Department of Defense (to SMW), Daniel F. and Ada L. Rice Foundation (to Wendy Rubinstein \& SMW) and Evanston Northwestern Healthcare (ENH) Breast/Ovarian Research Program (to XG).

\section{References}

I. Bartel DP: MicroRNAs: genomics, biogenesis, mechanism, and function. Cell 2004, I | 6:28I-297.

2. Lim LP, Lau NC, Garrett-Engele P, Grimson A, Schelter JM, Castle J, Bartel DP, Linsley PS, Johnson JM: Microarray analysis shows that some microRNAs downregulate large numbers of target mRNAs. Nature 2005, 433:769-773.

3. Lai EC, Tomancak P, Williams RW, Rubin GM: Computational identification of Drosophila microRNA genes. Genome Biol 2003, 4:R42.

4. Lim LP, Glasner ME, Yekta S, Burge CB, Bartel DP: Vertebrate microRNA genes. Science 2003, 299:1540.

5. Lim LP, Lau NC, Weinstein EG, Abdelhakim A, Yekta S, Rhoades MW, Burge CB, Bartel DP: The microRNAs of Caenorhabditis elegans. Genes Dev 2003, 17:991-1008.

6. Grad Y, Aach J, Hayes GD, Reinhart BJ, Church GM, Ruvkun G, Kim J: Computational and experimental identification of $C$. elegans microRNAs. Mol Cell 2003, II: I253-I263.

7. Berezikov E, Guryev V, van de B], Wienholds E, Plasterk RH, Cuppen E: Phylogenetic shadowing and computational identification of human microRNA genes. Cell 2005, I 20:2I-24.

8. Bentwich I, Avniel A, Karov Y, Aharonov R, Gilad S, Barad O, Barzilai A, Einat P, Einav U, Meiri E, Sharon E, Spector Y, Bentwich Z: Identification of hundreds of conserved and nonconserved human microRNAs. Nat Genet 2005, 37:766-770.

9. Sewer A, Paul N, Landgraf P, Aravin A, Pfeffer S, Brownstein MJ, Tuschl T, van Nimwegen E, Zavolan M: Identification of clustered microRNAs using an ab initio prediction method. BMC Bioinformatics 2005, 6:267.

10. Velculescu VE, Zhang L, Vogelstein B, Kinzler KW: Serial analysis of gene expression. Science 1995, 270:484-487.

II. Saha S, Sparks AB, Rago C, Akmaev V, Wang CJ, Vogelstein B, Kinzler $\mathrm{KW}$, Velculescu VE: Using the transcriptome to annotate the genome. Nat Biotechnol 2002, 20:508-5I2.

12. Chen J, Sun M, Lee S, Zhou G, Rowley JD, Wang SM: Identifying novel transcripts and novel genes in the human genome by using novel SAGE tags. Proc Natl Acad Sci USA 2002, 99:12257-12262.

13. Cai $\mathrm{X}$, Hagedorn $\mathrm{CH}$, Cullen R: Human microRNAs are processed from capped, polyadenylated transcripts that can also function as mRNAs. RNA 2004, 10:1957-1966.

14. Li SC, Pan CU, Lin WC: Bioinformatic discovery of microRNA precursors from human ESTs and introns. BMC Genomics 2006, 7:164.

15. Griffiths-Jones S, Grocock RJ, van Dongen S, Bateman A, Enright A): miRBase: microRNA sequences, targets and gene nomenclature. Nucleic Acids Res 2006, 34:D I 40-D I 44.

16. Barrett T, Suzek TO, Troup DB, Wilhite SE, Ngau WC, Ledoux P, Rudnev D, Lash AE, Fujibuchi W, Edgar R: NCBI GEO: mining mil- 
lions of expression profiles - database and tools. Nucleic Acids Res 2005, 33:D562-566.

17. Siddiqui AS, Khattra J, Delaney AD, Zhao Y, Astell C, Asano J, Babakaiff R, Barber S, Beland J, Bohacec S, Brown-John M, Chand S, Charest D, Charters AM, Cullum R, Dhalla N, Featherstone R, Gerhard DS, Hoffman B, Holt RA, Hou J, Kuo BY, Lee LL, Lee S, Leung D, Ma K, Matsuo C, Mayo M, McDonald H, Prabhu AL, Pandoh P, Riggins G], de Algara TR, Rupert JL, Smailus D, Stott J, Tsai M, Varhol R, Vrljicak P, Wong D, Wu MK, Xie YY, Yang G, Zhang I, Hirst M, Jones SJ, Helgason CD, Simpson EM, Hoodless PA, Marra MA: A mouse atlas of gene expression: large-scale digital gene-expression profiles from precisely defined developing C57BL/6] mouse tissues and cells. Proc Natl Acad Sci USA 2005, I02: I8485-18490.

18. Suh MR, Lee Y, Kim JY, Kim SK, Moon SH, Lee JY, Cha KY, Chung HM, Yoon HS, Moon SY, Kim VN, Kim KS: Human embryonic stem cells express a unique set of microRNAs. Dev Biol 2004, 270:488-498.

19. Lagos-Quintana M, Rauhut R, Yalcin A, Meyer J, Lendeckel W, Tuschl $\mathrm{T}$ : Identification of tissue-specific microRNAs from mouse. Curr Biol 2002, I 2:735-739.

20. Kent J: BLAT - the BLAST-like alignment tool. Genome Res 2002, I 2:656-664.

21. Wahl MB, Heinzmann U, Imai K: LongSAGE analysis revealed the presence of a large number of novel antisense genes in the mouse genome. Bioinformatics 2005, 21:1389-1892.

22. $\mathrm{Ge} X, W u \mathrm{Q}$, Jung $Y C$, Chen J, Wang SM: A large quantity of novel human antisense transcripts detected by LongSAGE. Bioinformatics 2006, 22:2475-2479.

Publish with Bio Med Central and every scientist can read your work free of charge

"BioMed Central will be the most significant development for disseminating the results of biomedical research in our lifetime. "

Sir Paul Nurse, Cancer Research UK

Your research papers will be:

- available free of charge to the entire biomedical community

- peer reviewed and published immediately upon acceptance

- cited in PubMed and archived on PubMed Central

- yours - you keep the copyright

Submit your manuscript here:

http://www.biomedcentral.com/info/publishing_adv.asp
BiolMedcentral 